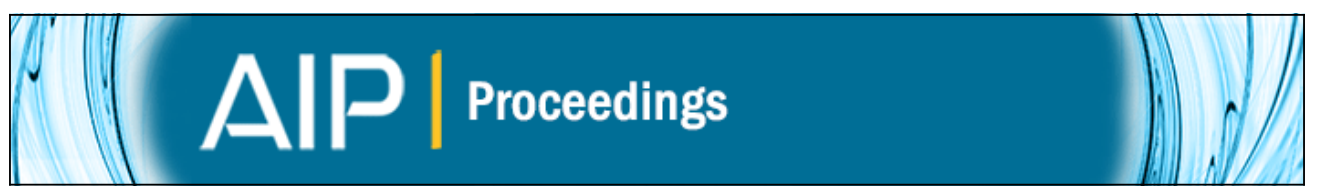

Limits of Perturbative Nonlinear Light Curve Analyses: the Case of G117-B15A

M. H. Montgomery, J. J. Hermes, and D. E. Winget

Citation: AIP Conference Proceedings 1273, 512 (2010); doi: 10.1063/1.3527877

View online: http://dx.doi.org/10.1063/1.3527877

View Table of Contents:

http://scitation.aip.org/content/aip/proceeding/aipcp/1273?ver=pdfcov

Published by the AIP Publishing

Articles you may be interested in

Exoplanets around G-K Giants

AIP Conf. Proc. 1331, 79 (2011); 10.1063/1.3556186

GD 358: The Case for Oblique Pulsation and Temperature Change

AIP Conf. Proc. 1273, 530 (2010); 10.1063/1.3527882

The Challenge of Explaining the Nonlinear Features in the Light Curve of the ZZ Ceti Star G117-B15A

AIP Conf. Proc. 1273, 508 (2010); 10.1063/1.3527876

The Problem of the Flickering Activity of the Recurrent Nova T CrB

AIP Conf. Proc. 637, 328 (2002); 10.1063/1.1518225

Magnetic field limit on SGR $1900+14$

AIP Conf. Proc. 599, 906 (2001); 10.1063/1.1434771 


\title{
Limits of Perturbative Nonlinear Light Curve Analyses: the Case of G117-B15A
}

\author{
M. H. Montgomery, J. J. Hermes and D. E. Winget \\ Department of Astronomy and McDonald Observatory, University of Texas, Austin, TX, USA
}

\begin{abstract}
This poster examines the nonlinear combination frequencies found in the DAV G117B15A. Using multiple epochs of observations we show that the ratio of the amplitude of the combination frequencies to those of the parent frequencies is not constant in time but rather varies on a time scale of years. This variation is difficult to explain in any model in which the modeled nonlinearities are second order, such as that of Brassard et al. [2], which accounts for these nonlinearities via the conversion from temperature variations to flux variations. In contrast, due to its extreme sensitivity, the convective light curve model can accomodate these variations with only modest variations in parameters such as $T_{\text {eff. }}$.
\end{abstract}

Keywords: convection — stars: oscillations — stars: variables: general — stars: individual (G117B15A)

PACS: $97.20 . R p, 97.30 .-b$

\section{ASTROPHYSICAL CONTEXT}

Combination frequencies are commonly found in the Fourier transforms of pulsating white dwarfs. These frequencies are presumably of a nonlinear origin and contain additional information about the pulsations and the structure of the star. Two major attempts have been made to model these nonlinearities. The first, by Brickhill [1], includes the interaction and feedback between the pulsations and the convection zone of the star. The second, by Brassard et al. [2], considers the nonlinearities to be due to the nonlinear relation between temperature change and the visible flux emitted.

The model by Brickhill has subsequently been expounded upon by others $[3,4,5$, $6,7]$. In its current formulation, the photospheric flux leaving the top of the convection zone is related to the flux entering it at the base of the convection zone, by

$$
F_{\text {phot }}=F_{\text {base }}+\tau_{C} \frac{d F_{\text {phot }}}{d t}
$$

where $\tau_{C} \propto T_{\text {eff }}^{-N}$ is a timescale associated with energy absorption and emission by the convection zone. It is temperature dependent since the size of the convection zone can vary greatly during a pulsation cycle. From simple mixing length theories we find that the exponent $N$ has a value of approximately 90 for DAVs and 23 for DBVs. Thus, even temperature variations of a few percent can potentially introduce large nonlinearities.

An alternate approach is to assume the temperature perturbations are fundamental (and therefore sinusoidal). In this spirit, Brassard et al. developed expressions for the flux nonlinearities in given filter bands accurate to second and third order in the perturbations. For cases of interest, the combination amplitudes generated were fairly small, such that the second order and third order expressions should be quite accurate. 


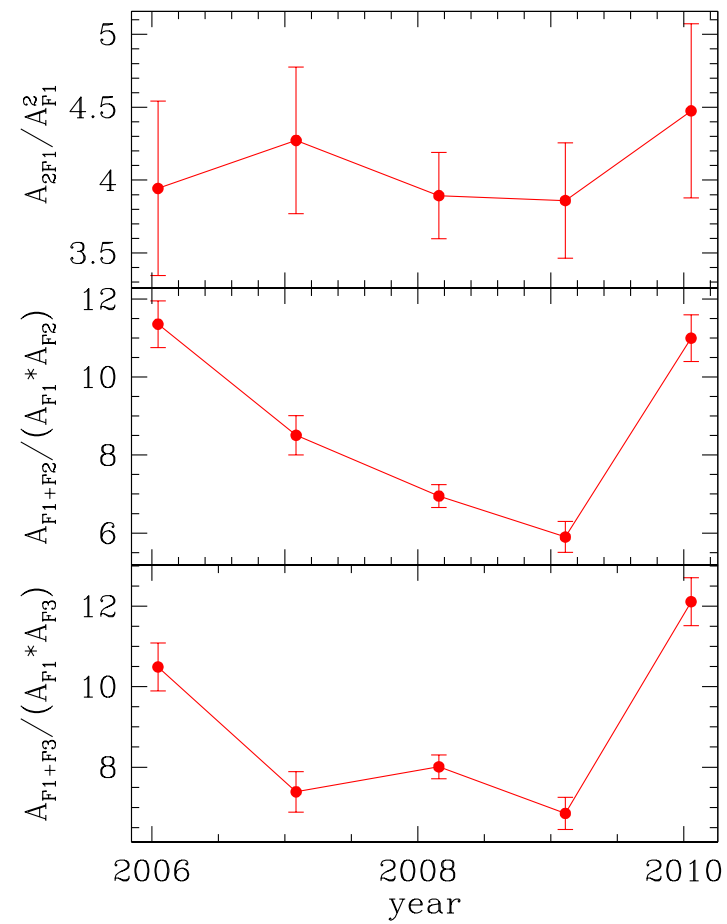

FIGURE 1. Relative combination frequency amplitudes in G117-B15A as a function of time. If the nonlinear mechanism were predominantly second order and constant in time then the above ratios would also be constant in time. Note, the modes labeled by F1, F2, and F3 have periods of 215.2, 270.5, and 304.1 seconds, respectively.

We note that $\mathrm{Wu}[5]$ also developed analytic formulae for the amplitudes of the combination frequencies accurate to second order. This approach was based on an analytical version of the physical model of Brickhill.

\section{A CRUCIAL TEST}

We define the quantity $\mathrm{R}$ to be

$$
R \equiv \frac{A_{F 1+F 2}}{A_{F 1} A_{F 2}}
$$

where $A_{F 1+F 2}$ is the amplitude of the combination frequency $F 1+F 2$ and $A_{F 1}$ and $A_{F 2}$ are the amplitudes of the parent modes with frequencies $F 1$ and $F 2$, respectively. For any second order theory $R$ should be constant, independent of any changes in the amplitudes of $A_{F 1}$ or $A_{F 2}$.

As a test case, we consider the pulsations in the well studied DAV G117-B15A. Over several years we have obtained data with the same telescope, filter, and detector ["Argos" 8], thus minimizing the chance that changes in the star are due to differences in our 


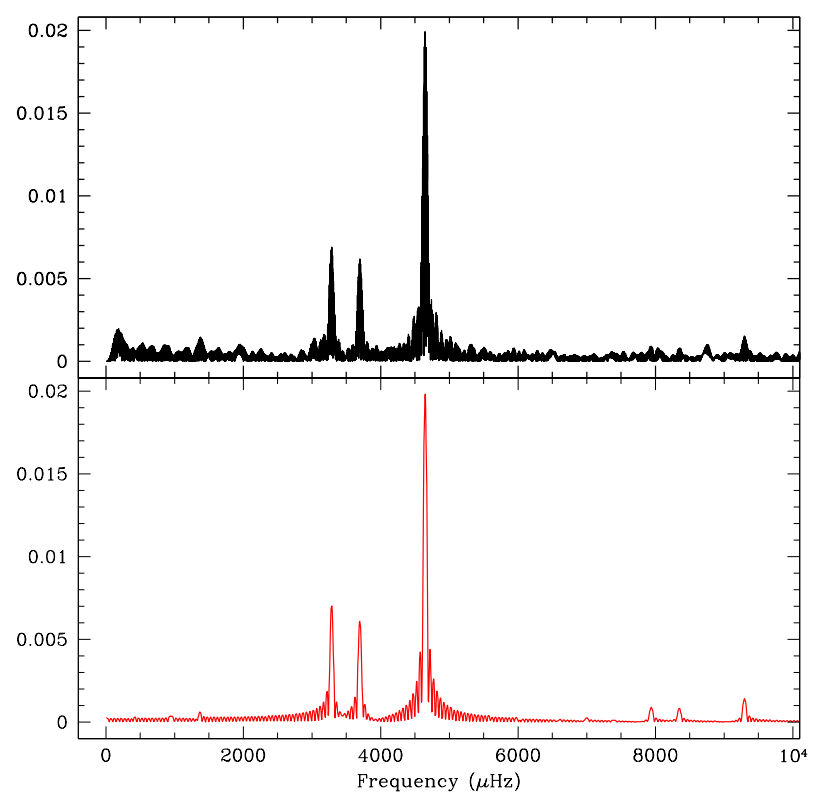

FIGURE 2. Comparison of observed fourier transform (top panel) and synthetic fourier transform based on the nonlinear light curve model.

observational setup. In Figure 1 we plot the value of $\mathrm{R}$ for the three largest combination frequencies for the years 2006 through 2010 . While the top panel is consistent with $\mathrm{R}$ constant in time, the middle and lower panels are not. These are very difficult to explain in the context of both the Brassard et al. and Wu models given the observed constancy of the temperature and luminosity of the star.

\section{NONLINEAR LIGHT CURVE FITS}

When $\tau_{0}$ is small we see from equation (1) that $F_{\text {phot }} \sim F_{\text {base }}$, so the convection zone introduces no nonlinearities. Thus, any nonlinearities will need to come from another source, i.e., the mechanism of Brassard et al. [2]. Such a mechanism is based on the nonlinear conversion between temperature and observed flux. Simplifying somewhat, $F \propto T^{\alpha_{V}}$, where $\alpha_{V} \sim 2-6$. This makes it clear why this nonlinearity is usually not dominant since it is much weaker than $\tau_{C} \propto T^{-90}$ for DAVs or $\tau_{C} \propto T^{-23}$ for the DBVs.

From direct fits to the light curves of G117-B15A we find that the value of $\tau_{0}$ is much smaller than it is for other DAVs. Equally good fits can be found assuming either $\ell=1$, $m=0$ or $\ell=1, m=1$ for all the modes, with values of $\tau_{0}$ ranging from $20 \mathrm{sec}$ to 40 sec. As shown in Figure 3, other stars for which we have made fits have values of $\tau_{0}$ larger than this. We have assumed $T_{\text {eff }}=11630 \mathrm{~K}$ for this star [9] but the pulsations seem to suggest that its actual temperature may be considerably hotter relative to the other variables in the strip. This would also make sense given that its short period pulsations would tend to place it near the blue edge of the instability strip rather than in the middle. 


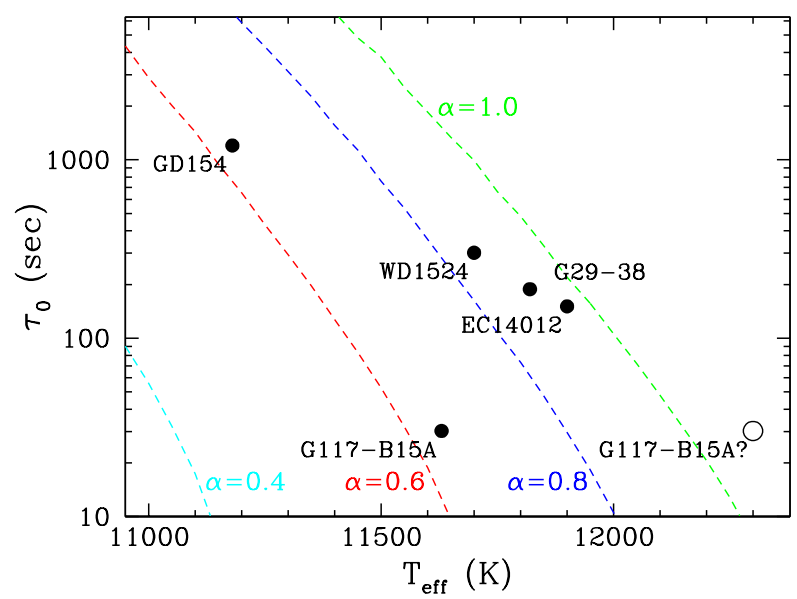

FIGURE 3. Determinations of $\tau_{0}$ versus $T_{\text {eff }}$ from nonlinear light curve fits. Our fits suggest G117G15A may have a higher $T_{\text {eff }}$ than that given in Bergeron et al. [9].

\section{CONCLUSIONS}

We have seen that the data on G117-B15A show that the amplitude ratio $\mathrm{R}$ of the combination and parent frequencies is not constant but varies with time well outside the error bars over a time scale of years. This is difficult to explain in any model in which the nonlinearities are second order. This poses a problem for the model of Brassard et al. [2] which accounts for these nonlinearities via the conversion from temperature variations to flux variations: large temperature variations in the equilibrium model would be required to change the nonlinear amplitudes. For the nonlinear light curve fitting model of Montgomery [7] these changes can be attributed to long time scale variations in the convection zone. This is at least plausible since the depth of the convection zone is a very sensitive function of the stellar parameters, so that a small change in the star's parameters (for instance) could lead to a measurable change in its convection zone.

\section{ACKNOWLEDGMENTS}

This work was supported by the National Science Foundation under grant AST-0909107, by the Norman Hackerman Advanced Research Program under grants 003658-02552007 and 003658-0252-2009, and by the Delaware Asteroseismic Research Center.

\section{REFERENCES}

1. A. J. Brickhill, MNRAS 246, 510 (1990).

2. P. Brassard, G. Fontaine, and F. Wesemael, ApJS 96, 545 (1995).

3. Y. Wu, and P. Goldreich, ApJ 519, 783 (1999).

4. P. Goldreich, and Y. Wu, ApJ 511, 904 (1999).

5. Y. Wu, MNRAS 323, 248 (2001).

6. J. Ising, and D. Koester, $A \& A$ 374, 116 (2001).

7. M. H. Montgomery, ApJ 633, 1142 (2005).

8. R. E. Nather, and A. S. Mukadam, ApJ 605, 846 (2004).

9. P. Bergeron, G. Fontaine, M. Billères, S. Boudreault, and E. M. Green, ApJ 600, 404 (2004). 